\title{
BMC Psychology reviewer acknowledgement 2015
}

Anna Clark

\section{Contributing reviewers}

The editors of BMC Psychology would like to thank all our reviewers who have contributed to the journal in Volume 3 (2015).

Richard Adams

The Netherlands

Eileen Anderson-Fye

USA

Lorenzo Avanzi

Italy

Daniel Baker

UK

Courtney Baker

USA

Baptiste Barbot

USA

Hendrik Berth

Germany

Ilse Blignault

Australia

India Bohanna

Australia

Chiara Buizza

Italy

Brendan Bunting

UK

Ana Butković

Croatia

Emanuele Antonio Maria Cappella Italy
Joseph Chilcot

UK

Mark Corrigan

Ireland

Georgina Cox

Australia

Kim De Jong

The Netherlands

Matty De Wit

The Netherlands

Dan Denis

UK

Theresa Dicke

Germany

Samantha Dockray

Ireland

Hongfei Du

China

Stefan Duschek

Austria

Andreas Eickhorst

Germany

William Ellison

USA

Cynthia Fair

USA
Katherine Fiori

USA

Signe Flottorp

Norway

Ted C. T. Fong

Hong Kong

David Frank

USA

Fabian Gander

Switzerland

David Garcia-Burgos

Switzerland

Abigail Gewirtz

USA

Emma Godfrey

UK

Francesca Graziano

Italy

Carla Groh

USA

Daniel Gruehn

USA

Aysegul Selcen Guler

Turkey

Kurt Hahlweg

Germany

Correspondence: anna.clark@biomedcentral.com

BioMed Central, Floor 6, 236 Gray's Inn Road, London WC1X 8HB, UK 
Åse Marie Hansen

Denmark

Phillipa Hay

Australia

Ashleigh Haynes

Australia

Sascha Hein

USA

Randall Holcombe

USA

Antje Horsch

Switzerland

Suzanne Huot

Canada

Sigurd William Hystad

Norway

Tomasz Jankowski

Poland

Aaron Jarden

New Zealand

Sameer Jauhar

UK

Josef Jenewein

Switzerland

Peter Johnston

UK

Sean Kang

USA

Natasha Khamisa

South Africa

Peter Kitchen

Canada

Dieter Kleinböhl

Germany

Heidi Koschwanez

New Zealand

Lenka Kostovicová

Slovakia

Emily Kothe

Australia

Kasia Kozlowska

Australia

Assaf Kron

Israel
Martin Lages

UK

Daniel Lakens

The Netherlands

Brian Lakey

USA

Marco Lauriola

Italy

Katharina Ledermann

Switzerland

Sakari Lemola

Switzerland

Gary Lewis

UK

Herman Hm Lo

Hong Kong

Elma Lorenzo-Blanco

USA

Vivian W. Q. Lou

Hong Kong

Sanja Lujic

Australia

Marta Marques

UK

Chantal Martin-Soelch

Switzerland

Barbara Maughan

UK

Peter Mckenna

Spain

Nadine Messerli

Switzerland

Lynn Miller

Canada

Mariella Miraglia

Canada

Paul Mkandawire

Canada

Martha Mohler

USA

Christine Moutier

USA

Kelly Lynn Mulvey

USA
Aja Murray

UK

Urs Nater

Germany

Nathalie Noret

UK

Sam Norton

UK

Fridtjof Nussbeck

Germany

Misari Oe

Japan

Claire O'Reilly

Australia

Nantje Otterpohl

Germany

Francesco Pagnini

Italy

Massimiliano Pastore

Italy

Nicola Petrocchi

Italy

Ron Prinz

USA

Diana Rancourt

USA

Tyler Renshaw

USA

Ian Robertson

UK

Anne Elizabeth Rogers

UK

Lucia Romo

France

Barry Rosenfeld

USA

Nina Rottmann

Denmark

Richard Rowe

UK

Kai Ruggeri

UK

Ulrich Schnyder

Switzerland 
Enrico Schulz

UK

Margaret Signorella

USA

Janne Skakon

Denmark

Andria Spyridou

Germany

Michael Stern

USA

Sarah Stewart-Brown

UK

Lisanne Stone

The Netherlands

Jade Thai

UK

Neil Thomas

Australia
Penny Trayner

UK

Linda Van Den Berg

Germany

Frank Van Der Horst

The Netherlands

Carol Van Hulle

USA

Michele Vecchione

Italy

Harrie Vorst

The Netherlands

Lisa Wagner

Switzerland

Marco Weber

Germany

Yvonne Whelan

UK
Redford Williams

USA

Daniel Wright

USA

Hongwei Xu

USA

Kimberly Young

USA

Robert Zachariae Denmark

Coralie Zumwald Switzerland

Laura Zwaan

The Netherlands 